\title{
Der für die Aufklärung des Patienten „verantwortliche“ Arzt
}

\author{
K. Wasserburg, Mainz
}

Wenn ein Patient ohne wirksame Einwilligung behandelt wird, begeht der Arzt u. U. durch die Behandlung eine rechtswidrige Körperverletzung. Die Einwilligung des Patienten in die Behandlung ist nur wirksam, wenn er zuvor vollständig aufgeklärt wurde. Das Problem der Vollständigkeit der Aufklärung wird in diesem Beitrag nicht behandelt. Die Frage, wer für die Aufklärung verantwortlich ist, kann bei der Behandlung durch mehrere Ärzte nicht immer zweifelsfrei beantwortet werden. Sie ist von großer Bedeutung für die ärztliche Praxis: Der Arzt, der für die „Behandlung“ des Patienten verantwortlich ist, ist auch für dessen Aufklärung verantwortlich.

Die Verantwortlichkeit für die Aufklärung ergibt sich daraus, daß der Arzt mit dem Patienten den Behandlungsvertrag schließt. Aus dem Arztvertrag hat der Arzt die Pflicht, den Patienten nach den Regeln der ärztlichen Kunst zu behandeln; daraus ergibt sich auch die Aufklärungspflicht.

1. Rechtswirksam aufklären kann allein ein Arzt, also z.B. keine Krankenschwester oder eine MTA, denn die Aufklärung ist Teil des „ärztlichen“ Gesprächs mit dem Patienten und damit Bestandteil der Heilbehandlung. Zwar wird durch den Betrieb der Krankenhäuser und Arztpraxen dem Arzt die Erfüllung der Aufklärungspflicht nicht immer leicht gemacht. Er ist in die Zusammenarbeit mit anderen Ärzten, dem Hilfspersonal und Apparaten eingegliedert und hat häufig nicht die nötige Zeit und Ruhe für die Gespräche mit dem Patienten. Das ändert aber nichts an der ihm grundsätzlich als ärztliche Aufgabe obliegenden „ärztlichen" Aufklärungspflicht. Ein medizinischer Laie kann, auch wenn er sich aufgrund jahrelanger einschlägiger Erfahrungen noch soviele Kenntnisse erworben hat, eine Aufklärung über die Risiken eines komplizierten Eingriffs schon grundsätzlich nicht leisten. Nichtärztliches Personal hat niemals die Legitimation und Kompetenz, Aufklärungsfragen zu übernehmen.

Der Patient kann mit einer Aufklärung durch Dritte einverstanden sein und dadurch dem Arzt gegenüber auf dessen ärztliche Aufklärung verzichten. Er trägt dann selbst das Risiko der unzureichenden Aufklärung. Der Aufklärungsverzicht ist m. E. rechtswirksam; der Patient kann auch pauschal ohne Aufklärung in die Behandlung an sich einwilligen.

2. Wird der Patient vom Hausarzt an einen Facharzt oder eine Klinik zur weiteren Behandlung über-

Akt. Urol. 21 (1990) 201-203
c) Georg Thieme Verlag Stuttgart · New York wiesen, wenn die Möglichkeiten des erstbehandelnden Arztes nicht ausreichen, dann sind sowohl der erstbehandelnde Arzt, als auch alle später behandelnden Ärzte selbständig für die Aufklärung des Patienten verantwortlich. Bei einer „Arztkette“ muß der jeweils behandelnde Arzt die Aufklärungsbedürftigkeit des Patienten feststellen und im Zweifel nochmals die gesamte Vollaufklärung wiederholen, es sei denn, der Patient verzichtet ganz oder teilweise darauf.

Bei der Zuständigkeit des Hausarztes für die Aufklärung ist danach zu unterscheiden, ob er seinen $\mathrm{Pa}$ tienten in eine Klinik zur weiteren Behandlung einweist, oder ob er den Patienten nach der Krankenhausbehandlung weiterbehandelt.

a) Im Regelfall wird der Patient vom Hausarzt in eine Klinik (oder zu einem anderen Arzt) zur weiteren Behandlung überwiesen. Dann geht mit der Übernahme der Behandlung durch die Klinik (oder den anderen Arzt) auch die Verantwortlichkeit für die Aufklärung auf den Übernehmenden über.

Hier hat der Arzt, auf den die Behandlung weiterübertragen wurde, die Pflicht festzustellen, ob und inwieweit der Patient bereits aufgeklärt wurde. Es genügt nach der Rechtsprechung des Bundesgerichtshofs für die Wirksamkeit der Einwilligung, wenn der Patient vor der Konsultation des ihn später behandelnden Arztes von anderen Ärzten ausreichend aufgeklärt wurde (1). Ein Klinikarzt kann nicht ohne Kontrolle einfach davon ausgehen, daß vom einweisenden Arzt schon eine ausreichende Aufklärung durchgeführt wurde. Es wäre nicht nur ein überflüssiger Aufwand, den Patienten, der vielleicht ausreichend aufgeklärt ist, nochmals vorsorglich aufzuklären; der Patient bedarf vor allem der Schonung, d.h., er soll nur im „notwendigen“ Umfang aufgeklärt werden und keine unnötige Doppelaufklärung erhalten. Wenn er im umgekehrten Fall nicht aufgeklärt wurde, kann er nicht einfach ohne Überprüfung behandelt werden, denn dann wäre die Einwilligung möglicherweise unwirksam. Die Notwendigkeit der Kontrolle folgt aus der ärztlichen Sorgfaltspflicht, die nicht nur den rein medizinischen Teil der Aufklärung betrifft, sondern auch die Aufklärung als Teil der ärztlichen Behandlung. Wird die Kontrolle unterlassen, so ergeben sich aus diesem Versäumnis selbst keine unmittelbaren Folgen für den Arzt, denn sie ist keine eigenständige Rechtspflicht. Wenn aber die Kontrolle nicht stattfindet, geht der behandelnde Arzt das Risiko ein, wegen der mangelhaften Einwilligung durch die Behandlung eine rechtswidrige Körperverletzung zu begehen. Der Patient kann sich bei unterlassener Kontrolle nicht auf ein Aufklärungsversäumnis (z. B. des Klinikarztes) berufen, wenn er bereits (etwa vom Hausarzt) aufgeklärt 
wurde, denn er hat in diesem Fall seine Einwilligung in Kenntnis aller relevanten Umstände gegeben (2). Liegt diese Ausnahme nicht vor, so hilft es dem Krankenhausarzt auch nicht in jedem Fall fehlerhafter oder unterlassener Aufklärung, daß die Klinikleitung die Organisation und Überwachung der Aufklärung übernommen hat (3).

Beim 52. Deutschen Juristentag 1978 in Wiesbaden waren einige Mediziner unter Hinweis auf organisatorische und zeitliche Probleme bei der Aufklärung der Meinung, daß die Aufklärung bereits durch den Hausarzt vorgenommen werden soll (4). Der Bundesgerichtshof hat nach dem Juristentag entschieden, daß der Hausarzt unter bestimmten Umständen für die Aufklärung verantwortlich sei. In diesem besonderen Fall hatte der Hausarzt dem Chefarzt der chirurgischen Abteilung die Appendektomie des Patienten „nicht nur zur Prüfung anheimgestellt, sondern sie kategorisch als erforderlich bezeichnet". Er tat das ohne jede Begründung und ohne Befundhinweis. Der Hausarzt ging offensichtlich davon aus oder rechnete damit, da 3 der Chefarzt seiner Auffassung ohne weiteres folgen würde. Dann aber hatte nach Ansicht des Bundesgerichtshofs ,in erster Linie" schon den Hausarzt die Pflicht getroffen, mit dem Patienten vor Abfassung eines Überweisungsschreibens das Für und Wider einer Operation unter entsprechender Aufklärung über deren Risiko zu erörtern (5).

Diese Entscheidung wurde in der juristischen Literatur stark kritisiert, vor allem wegen der Überforderung des Hausarztes durch die zu sehr „ausgeweitete“ Aufklärungspflicht (6).

Der Kritik ist entgegenzuhalten, daß der Hausarzt zur Aufklärung überhaupt nur im Rahmen seiner Möglichkeiten verpflichtet sein kann. Auch vom Hausarzt kann nichts Unmögliches verlangt werden. Im Fall des Bundesgerichtshofs hatte der Hausarzt sich durch sein Verhalten gleichsam an die Stelle des Klinikarztes gestellt und offenbar jede weitere Kontrolle seines Befundes für überflüssig gehalten. Wenn er sich aber so verhält, muß er auch in diesem Sonderfall die Aufklärungskonsequenzen tragen, aber auch nur dann. Die Garantenstellung des Hausarztes darf nicht überspannt werden. So ist z. B. der Hausarzt nicht verpflichtet, dem Patienten zu sagen, er müsse den später behandelnden Arzt auf die besondere Dringlichkeit seines Falles hinweisen. Was im Ernstfall zu tun ist, hat allein der später behandelnde Facharzt zu beurteilen. Der einweisende Hausarzt ist $m$. E. weder verpflichtet noch in der Lage, durch weitergehende Belehrungen allen denkbaren Eventualitäten einer späteren Behandlungsbedürftigkeit des Patienten Rechnung zu tragen. Gerade bei besonders schwierigen Eingriffen, die dem Patienten eine sehr schwere Entscheidung abverlangen, erfordert es das Vertrauensverhältnis zwischen Arzt und Patienten, daß der den Eingriff vornehmende Chirurg das Aufklärungsgespräch führt. Das aber kann der Hausarzt gar nicht leisten.

b) Nach der Klinikbehandlung muß der Hausarzt den Patienten über das weitere Vorgehen, aber auch über Besonderheiten, z. B. über eine fehlerhafte Klinikberatung, informieren. Im Verhältnis zwischen Hausarzt und Klinikarzt gilt zwar der Vertrauensgrundsatz, nach dem der Hausarzt auf die bessere Sachkunde und größere Sachnähe der Klinikärzte vertrauen darf. Dieser Grundsatz kommt aber nur soweit zur Anwendung, als nicht ernste Zweifel an der Richtigkeit der Krankenhausbehandlung und der dort dem Patienten gegebenen ärztlichen Ratschläge bestehen.

3. Wird der Patient dagegen in der Klinik von mehreren Ärzten behandelt, so stellt sich die Aufklärungsfrage zunächst hauptsächlich als Organisationsfrage dar. Der in diesem Fall für die Aufklärung verantwortliche Arzt, meist der Chefarzt, muß bei der Übertragung der Aufklärung auf einen anderen Arzt, wenn er nicht selbst aufklärt, dafür sorgen und überprüfen, daß der Patient ordnungsgemäß aufgeklärt wird. Wenn die Organisation der Aufklärung in der Klinik nicht funktioniert, der Arzt infolgedessen den Patienten ohne wirksame Einwilligung behandelt, liegt eine rechtswidrige Körperverletzung vor, die er zu verantworten hat, sofern keine besonderen entlastenden Umstände im Einzelfall vorliegen.

Eine sachgerechte Aufklärung des Patienten wird durch die zunehmende Arbeitsteilung in der Medizin immer mehr erschwert, weil die Gefahr besteht, daß der einzelne Arzt dabei den Überblick verliert, und außerdem, daß sich einer auf den anderen verläßt. Hier geht es nicht nur um das Problem, wie weit der Arzt für Fehler anderer an der Behandlung beteiligter Personen verantwortlich ist, sondern um die Gewährleistung der Aufklärung. Häufig wird der Patient nicht nur im Rahmen der Gesamtbehandlung, sondern auch innerhalb eines Behandlungsabschnittes von mehreren Ärzten, nicht nur im Krankenhaus, sondern auch bei niedergelassenen Ärzten, behandelt: - bei niedergelassenen Ärzten nimmt die Zusammenarbeit seit längerem deutlich zu (Gemeinschaftspraxis, Praxisgemeinschaft usw.). Ambulantes Operieren ist ein weiteres aktuelles Beispiel für ärztliche Zusammenarbeit. Damit gewinnt die Frage der Delegierbarkeit der Aufklärung über den Krankenhausbereich hinaus an Bedeutung.

a) Der verantwortliche Arzt muß den Patienten nicht persönlich aufklären, wenn dieser nicht darauf besteht, sondern er kann die Aufklärung an einen anderen Arzt delegieren. Das betrifft aber nur die Durchführung der Aufklärung; verantwortlich für die ordnungsgemäße Aufklärung des Patienten ist - neben dem mit der Aufklärung beauftragten Arzt - nach wie vor der delegierende Arzt (z. B. der Chefarzt), wenn er den Patienten weiterhin behandelt; daher muß er die Durchführung der Aufklärung kontrollieren und überwachen.

b) Auch die Klinikleitung hat die Durchführung der Aufklärung zu überwachen. Mit dieser Tatsache allein kann sich der Arzt aber nicht ohne weiteres wirksam verteidigen; die Frage kann jedoch im Rahmen des Irrtums oder der Strafzumessung von Bedeutung sein. Nur wenn im Krankenhaus im Rahmen der Arbeitsteilung die Aufklärung des Patienten besonders geregelt ist und überwacht wird, kann sich der den Eingriff vornehmende Arzt darauf verlassen, daß die sich über einen längeren Zeitraum hinweg bewährte Regelung auch im Einzelfall bewährt. Er kann in diesem Fall davon ausgehen, daß der Patient wirksam in die Operation eingewilligt hat. Es ist Tatfrage, inwieweit dem Arzt ein Irrtum vorwerfbar ist, wenn dadurch keine Aufklärung stattfand. 
Die Leitungs- und Aufsichtspflicht, die dem Vorstand der Klinik obliegt, bezieht sich auch darauf, daß im Krankenhausbetrieb die ärztliche Aufklärung beachtet und ihren Erfordernissen Genüge getan wird (7). Der Bundesgerichtshof betont, es liege zwar auch im eigenverantwortlichen Ermessen der behandelnden Ärzte, darüber zu befinden, welche therapeutischen Mittel zur Anwendung gebracht werden sollen. Die Krankenhausleitung müsse sich aber in geeigneter Weise vergewissern, daß z.B. auch bei medikamentöser Behandlung eine notwendige Aufklärung der Patienten nicht versäumt werde.

„Was der Krankenhausvorstand tun muß, um seiner Leitungs- und Aufsichtspflicht in dieser Hinsicht zu genügen, läßt sich nicht allgemein sagen; es hängt von den jeweiligen Verhältnissen des betreffenden Krankenhauses, der Größe und Ordnung des Krankenhausbetriebes, der personellen Zusammensetzung des ärztlichen Dienstes usw. ab, durch welche Maßnahmen die Krankenhausleitung dafür zu sorgen hat, daß die beim Krankenhaus tätigen Ärzte der Notwendigkeit ärztlicher Aufklärung gerecht werden." (8)

Bei größeren Kliniken ist es gar nicht möglich, daß die Klinikleitung in jedem Fall persönlich wegen der Zustimmung zu einer Operation mit den Angehörigen verhandelt. Wenn der Leiter der Klinik aber dazu persönlich nicht in der Lage ist, muß er wenigstens Anordnungen treffen, die zuverlässige Gewähr dafür bieten, da $\beta$ z.B. die Einwilligung des gesetzlichen Vertreters eines zu operierenden Kindes zuvor eingeholt wird.

Die Unsicherheiten im Einzelfall, die sich trotz sorgfältigster Organisation nicht vermeiden lassen, kennt jeder Arzt - er muß damit rechnen, daß die Organisation im Einzelfall versagen kann. Schon deswegen entbindet ihn die Organisationspflicht der Klinikleitung nicht von der Pflicht, vor der Behandlung die Aufklärungsbedürftigkeit des Patienten festzustellen.

Der Bundesgerichtshof verlangt hauptsächlich klare Kompetenzen und eine funktionierende Organisation: „Gerade in Fällen einer ärztlichen Arbeitsteilung muß sichergestellt sein, daß notwendige Maßnahmen, zu denen auch die ärztliche Aufklärung gehört, nicht deswegen unterbleiben, weil sich einer auf den anderen verläßt, ohne daß die Kompetenzen geklärt und Fehlerquellen organisatorisch soweit als möglich ausgeschaltet sind." (9) Nur einer praktikablen, d.h. nicht uferlosen Kontrollpflicht kann der Krankenhausarzt nachkommen. Wenn die Organisation der Aufklärung im Einzelfall einmal nicht funktioniert, kann er dafür ohne das Hinzukommen besonderer Umstände nicht verantwortlich sein. Außerdem muß von den meisten Patienten eine gewisse zumutbare Mitwirkung durch das Stellen entsprechender Fragen erwartet werden.

\section{Literatur}

1 BGH NJW 1961, 2203; BGH NJW 1980, 633; BGH NJW 1981, $1319 f$.

2 So zu Recht Giesen, JZ 1982, 345, 398; BGH VersR 1979, 720f.; OLG Stuttgart, VersR 1981, 342f,; BGH NJW 1980, 1905; BGH NJW 1983, 2642; Schünemann, NJW 1980, 2753

3 Dazu weiter unten

4 Weissauer, in: v. Falkenhausen, JZ 1978, $767 \mathrm{f}$.

${ }^{5}$ BGH NJW 1980, 633f.; kritisch dazu Hirsch, LK, § 226 a Anm. 21

6 Giesen, JZ 1982, 345, 395; Hirsch, LK, \$ 226 a Anm. 21. Schünemann, NJW 1980, 2753; Tempel, NJW 1980, 615; Wachsmuth, JR $1981,19,21 \mathrm{f}$.

7 BGH NJW 1956, 1106; BGH NJW 1963, 393, 395

8 BGH NJW 1963, 393, 395

9 OLG Celle, NJW 1979, 1251 f. mit kritischer Anm. Wachsmuth

Dr. K. Wasserburg

Holzstraße 42

D-6500 Mainz 